\title{
Nueva edición crítica de El lugar sin límites de José Donoso en Colección Biblioteca Chilena: notas en torno a un proyecto de edición
}

\author{
María Laura Bocaz Leiva \\ University of Mary Washington \\ mbocazle@umw.edu
}

\begin{abstract}
RECEPCIÓN: 06/12/2019
\end{abstract}
ACEPTACIÓN: 24/01/2019

$\mathrm{H}$ e tenido el honor y el placer de editar, para Colección Biblioteca Chilena de Ediciones Universidad Alberto Hurtado, esta novela de José Donoso (19241996) que, a más de cincuenta años de su primera edición en México (1966), constituye tanto una de las principales novelas del escritor chileno como un hito dentro de la literatura latinoamericana. Agradezco al comité editorial de (an)ecdótica, especialmente a Ana Laura Zavala Díaz, que me invitara a participar del diálogo centrado en la labor ecdótica de textos con el que la revista contribuye, llenando desde su primer número un vacío indiscutible en nuestro campo al proveer esta instancia de reflexión y colaboración en torno al quehacer de rescate y fijación de textos iberoamericanos.

Inicio esta nota resaltando el nexo de la novela con México, para luego comentar brevemente los contenidos y detenerme en algunos detalles surgidos a partir del trabajo ecdótico, así como en el lugar que le dimos a los materiales de archivo del escritor en este volumen.

\section{El lugar sin límites y su vínculo intrínseco con México}

La conexión de El lugar sin límites con México desde su génesis fue destacada por el propio Donoso en su célebre Historia personal del "boom". En su recuento, hoy uno de los principales testimonios de este importante periodo de la historia literaria y cultural de América Latina, Donoso señala que escribió la novela en "el pabellón que Carlos Fuentes" les alquiló a su esposa María Pilar y a él, "en el fondo de su jardín en la calle Galeana" (114). A juzgar por sus palabras, ambos escritores, quienes - se reconocieron tan sólo dos años antes en el Congreso de Intelectuales celebrado en Concepción en 1962 - realizaban al unísono dos de las principales novelas de la nueva narrativa del Boom: "Yo tecleaba metido en la sombra del pabellón del fondo del jardín. 
Al otro lado, en la casa grande, con Las estaciones de Vivaldi puesto a todo lo que daba el tocadiscos, Carlos Fuentes escribía Cambio de piel' (115). ${ }^{1}$

Tanto las anotaciones en los diarios de escritura que guardan las huellas del proceso creativo de la novela como las versiones mecanografiadas implicadas en el desarrollo composicional de El lugar sin límites confirman que la totalidad de la redacción de ésta - la segunda novela de Donoso-, efectivamente, se realizó en México. El autor solía especificar, por lo menos hasta la década del setenta (no he estudiado los materiales de escritura posteriores a la publicación de El obsceno pájaro de la noche), los lugares y años relacionados con el proceso creativo de sus textos. En la última página de la copia del mecanoescrito más tardío de la novela, archivado en la Universidad de Iowa, se lee:

$\begin{array}{ll}\text { Cerrada de Galeana } 16 & \text { Diciembre } 1964 \\ \text { San Ángel Inn } & \text { Febrero } 1965 \\ \text { México D. F. } & \end{array}$

El impacto de Fuentes en la escritura de la novela no se limita, sin embargo, a haberle facilitado al autor chileno un espacio para la ejecución de su obra. En su Historia personal, Donoso también recuerda cómo gracias al escritor mexicano publicó la novela con la editorial Joaquín Mortiz en vez de con Zig-Zag, con el objetivo de pagar la deuda contraída anteriormente con esa casa:

Fuentes opinó que era demasiado bueno para usarlo con el fin de saldar una absurda deuda de mil dólares en Chile, ya que jamás saldría de allí, puesto que el monopolio editorial lo impediría. Que publicara ese libro, más bien, en México, donde mi obra quedaría más expuesta. La Editorial Joaquín Mortiz, entonces flamante y activa, se interesaba (120).

Las notas en tinta roja y de caligrafía apurada que ocupan la página 114 del diario 32 de la colección en Iowa revelan que, para el 28 de febrero de 1965, la novela ya había sido "leída y aprobada x Carlos Fuentes", así como "entregada a Joaquín Díez-Canedo". Cabe destacar que con la inclusión de El lugar sin límites en la prestigiosa serie "Del volador" en 1966, gracias a la influencia de Fuentes, Donoso no sólo publicó por primera vez fuera de Chile, sino también en plena efervescencia del Boom latinoamericano y en una de las principales casas editoriales del mundo hispanohablante.

Este vínculo intrínseco de El lugar sin límites con México no se restringe a la edición princeps en "una de las altas cumbres de la inventiva editorial en México" (Mejía: 4);

${ }^{1}$ Carlos Fuentes y José Donoso coincidieron en el colegio The Grange cuando el padre de Fuentes, diplomático, había sido comisionado a Chile (Donoso, 1998: 58). Si bien Donoso no se graduó de esta institución, The Grange recibió una donación de parte del autor chileno, que contenía, además de una selección de su biblioteca personal, un par de fotografias y otros objetos personales. En 1996, The Grange honró al escritor con un edificio, el José Donoso Center. 
se reitera a fines de los setenta, cuando en 1978 la novela llega al cine de la mano de uno de sus principales cineastas: Arturo Ripstein. ${ }^{2}$ Un último detalle interesante que habla de la relación de El lugar sin limites con México surge del cotejo de las diferentes copias materiales estudiadas para el establecimiento del texto. Todo indica que, cuando El lugar sin límites se vuelve a editar en América Latina, no son las ediciones españolas -Euros (1975), Sedmay (1977), Bruguera (1977) - las que conforman la base de ese texto que, nuevamente, se fija y se pone en circulación, sino la primera, la mexicana, y que, cuando la novela se edita otra vez en España en 1979, la versión de Seix Barral no se apoya en las ediciones hechas "en casa", por lo contrario, utiliza la edición princeps. ${ }^{3}$

\section{Contenidos del volumen}

La edición comienza con una "Introducción" que gira en torno a la obra de José Donoso, elaborada a partir de múltiples fuentes y testimonios, entre los que se incluye la correspondencia personal del escritor. El recorrido inicia con la publicación de sus primeros dos cuentos "The Blue Woman" (1950) y "The Poissoned Pastries" (1951) en la revista MSS. ${ }^{4}$ Además de ofrecer una revisión de su producción narrativa, con énfasis en El lugar sin límites, se documenta el trabajo de Donoso en la revista Ercilla, con la compañía de teatro ICTUs y en el taller para escritores que por años llevó a cabo en su casa. Este apartado se cierra con la mención de algunos de los múltiples reconocimientos que le fueron otorgados, entre ellos el tardío Premio Nacional de Literatura en 1990, así como con una alusión a la publicación de textos en forma póstuma.

Anteceden a la novela anotada una sección dedicada a la "Historia del texto" y otra a los "Criterios editoriales". La primera parte ofrece una revisión de la edición princeps, para luego detenerse en hitos importantes de la circulación de la novela, tales como su llegada al ámbito editorial español en el ocaso de la dictadura de Franco, su publicación en 1979 en una Seix Barral que ya no contaba con Carlos Barral en sus filas, y el retorno de la novela al mercado latinoamericano en 1990. En el apartado relativo a los criterios editoriales se especifican las normas que rigen la edición.

\footnotetext{
${ }^{2}$ El guion estuvo a cargo de Manuel Puig, quien finalmente prefirió no aparecer como autor. Josefina Delgado, en "La mirada sin cuerpo", aclara por qué el escritor argentino insistió en que "su nombre no figurara". El motivo fue la posibilidad de que censuraran la película tras el cambio del director del Banco Nacional Cinematográfico (25).

${ }^{3}$ La primera edición en Seix Barral es de 1979. El cotejo de las novelas publicadas posteriormente (1981, 1985 y 1987) demuestra que se trata de reimpresiones sucesivas de esa primera edición, que es idéntica a la princeps.

${ }^{4}$ Revista publicada por los estudiantes de la Universidad de Princeton, coeditada por José Donoso y Robert V. Keeley entre 1949 y 1951.
} 
En sintonía con los cinco volúmenes que en Colección Biblioteca Chilena preceden a esta edición de El lugar sin limites, ${ }^{5}$ el tomo también incluye una selección de lecturas críticas claves que constituyen hitos en la historia de la recepción de la novela, la cual se inaugura de manera temprana con dos artículos en 1967: "El mundo de José Donoso" de Emir Rodríguez Monegal, publicado originalmente en la revista Mundo Nuevo, y "El infierno de la ambigüedad" de Alejandro Patemain, que aparece en la revista uruguaya Temas. El dossier, no obstante, se abre con los dos artículos que han marcado por décadas la recepción crítica de la novela: el de Severo Sarduy, "Escritura/travestismo" (1968), y el de Fernando Moreno, "La inversión como norma. A propósito de El lugar sin límites" (1975). El tercer texto del dossier presenta por primera vez en español, gracias a la traducción de la Dra. Ángela San Martín Vásquez, el capítulo que a inicios de los noventa la Dra. Sharon Magnarelli dedicó a El lugar sin límites en su libro angular Understanding José Donoso (1993). El estudio de Magnarelli — una de las principales especialistas en la obra del chileno - es uno de los primeros en dar a la cuestión del género un lugar protagónico en la lectura de la novela, así como en problematizar el pronombre que se emplea para aludir a la Manuela: "A lo largo de este capítulo me refiero a Manuela con pronombres que simultáneamente evocan ambos géneros - él/ella - para enfatizar la dualidad del personaje, la cual es fundamental para el mensaje de Donoso, y para evitar privilegiar ya sea la faceta masculina o femenina de su caracterización”. ${ }^{6}$

La incorporación de este texto de importancia histórica dentro de la recepción crítica de la novela permite al lector ver cómo a partir de los años noventa se genera, de manera paulatina, una significativa vuelta de tuerca en la forma de leer la obra a la luz de los estudios de género y sexualidades, ofreciendo nuevas zonas de interpretación y proponiendo lecturas radicalmente diferentes de, por ejemplo, la noche de la apuesta, la identidad de la Manuela y la violencia ejercida en su contra, entre otros. ${ }^{7}$ Cierran el dossier dos ensayos emblemáticos de la recepción crítica de la novela en el siglo xxi: "Espacio y sexualidad en El lugar sin límites de José Donoso" de Andrea Ostrov (2014) y "José Donoso y las masculinidades monstruosas de la reforma agraria chilena" (2016) de Carl Fischer. Este último, traducido al español por el propio autor,

${ }^{5}$ Baldomero Lillo, Obra completa (edición de Ignacio Álvarez y Hugo Bello), fosé Victorino Lastarria (edición de Hugo Bello), Marta Brunet (tomos I y II, edición de Natalia Cisterna) y El roto (edición de Osvaldo Carvajal). Véase: http://ediciones.uahurtado.cl/coleccion/biblioteca-chilena/

${ }^{6}$ Traducción al español de San Martín Vásquez de la nota aclaratoria de Magnarelli en la quinta nota del capítulo.

${ }^{7}$ El único estudio de relevancia histórica que, por motivos de fuerza mayor, no pudimos incluir en el dossier es el de Ben Sifuentes Jáuregui, "Gender without Limits: Transvestism and Subjectivity in El lugar sin limites". Este texto fue publicado en Sex and Sexuality in Latin America (1997), editado por Daniel Balderston y Donna J. Guy; disponible en su traducción al español (Sexo y sexualidades en América Latina) desde 1998 en la editorial Paidós.

134 (an)ecdótica * vol. IV, núm. 2, julio-diciembre 2020 
plantea una lectura queer de la novela como "uno de los principales artefactos culturales en los que los atributos discursivamente patriarcales de la modernización agraria chilena se manifiestan y se disputan de manera más patente" (289).

El volumen se cierra con una cronología y una bibliografía elaboradas en colaboración con Daniela Buksdorf Krumenaker. La primera ofrece datos sobre la vida y obra de Donoso, el Boom latinoamericano, Chile, Latinoamérica y el mundo, con la finalidad de poner en relación los principales hitos bio-bibliográficos de Donoso con los más significativos de la historia social e intelectual del periodo, que coinciden con la vida y obra del escritor. La bibliografía de la obra reúne los estudios que conforman su recepción crítica.

\section{Algunos detalles en relación con el establecimiento del texto}

Para la fijación y establecimiento del texto se tomaron dos ediciones: la que creemos fue la última supervisada por el escritor (primera edición de Alfaguara en 1995) y la princeps. Las variantes que arrojó el cotejo de ambas son escasas. Se observan enmiendas de erratas presentes en la princeps, del tipo "rio" por "rió". También se encontraron modificaciones en la puntuación, tales como el cambio de punto y seguido por punto y aparte, y eliminación o inserción de comas, por ejemplo: "oyó, la bocina en la otra calle frente al correo" (Mortiz: 9) versus "oyó la bocina en la otra calle frente al correo" (Alfaguara: 11). Permutas léxicas, como "despacito" (Mortiz: 14) por "despacio" (Alfaguara: 16). Uso de cursivas, comillas y también modificaciones en el número de caracteres empleados en vocablos, en los que se representa alargamiento mediante la reiteración de la vocal. Por ejemplo, en la princeps se cuentan ocho vocales "a" en "vereda" y trece en "tropical", las últimas dos separadas por un guion:

"Veredaaaaaaaa tropicaaaaaaaaaaa-aal” (17).

En tanto que en la edición de Alfaguara aparecen siete "a" en "vereda" y diez en "tropical", sin uso de guion:

"Veredaaaaaaa
tropicaaaaaaaaaaal" (18).

En este caso, optamos por mantener el número de vocales y el guion observable tanto en la edición princeps como en los borradores mecanografiados de la novela.

En la misma línea, decidimos restablecer el complemento directo "mis manos", omitido en la edición de Alfaguara, tomando como punto de partida que esta elisión no aparece en la edición princeps ni en ninguna de las versiones mecanografiadas exis- 
tentes de la novela: "hasta que me mire [la Manuela] con esos ojos de redoma aterrados y hundiendo mis manos en sus vísceras babosas y calientes para jugar con ellas, dejarla allí tendida, inofensiva, muerta: una cosa" (Mortiz: 127).

Antes de cerrar esta sección, quisiera detenerme en dos elementos particularmente interesantes surgidos durante el cotejo de la edición princeps con las tres primeras ediciones de la novela en España. El primero tiene que ver con la ausencia de tildes en las mayúsculas en las ediciones españolas. Este detalle, que puede parece nimio, es una huella significativa de la ventaja con la que contaba el campo editorial mexicano en los años setenta en comparación con el español. Al respecto, Eduardo Mejía advierte que las editoriales mexicanas a mediados de esa década, es decir, casi diez años antes que las españolas, estaban en condiciones de tildar mayúsculas (6).

El segundo corresponde a las intromisiones editoriales más relevantes, identificadas durante el trabajo ecdótico, que se observan en lo que he llamado el "primer ciclo español" (para diferenciarlo de la impresión de la novela a partir de 1979 en Seix Barral). Una de las más destacadas implica un cambio de vocablo ("agrandaría" por "agradaría"), que afecta sustancialmente el contenido semántico del enunciado atribuible a la Japonesa Grande. En la edición princeps, la dueña del burdel sueña con ampliar el prostíbulo tras ganarle al terrateniente Cruz la apuesta, superando a su competencia, el burdel de la Pecho de Palo (las negritas en ambos enunciados son mías): "Me agrandaría y mi casa sería más famosa que la de la Pecho de Palo" (Mortiz: 74). En la edición de Bruguera, mediante el uso del verbo "agradar", se elimina el impacto que tendría ganar la apuesta para el burdel y se lo circunscribe a la Japonesa Grande: "Me agradaría y mi casa sería más famosa que la de la Pecho de Palo" (98). Concluyo esta sección con la intromisión editorial más dramática: el reemplazo de "Japonesa", en "don Alejo y la Japonesa chocaron sus vasos llenos" (Mortiz: 83), por "Japonesita": "don Alejo y la Japonesita chocaron sus vasos llenos" (Bruguera: 111). En este último la intervención editorial resulta descabellada, ya que el personaje Japonesita no ha nacido para el momento en el que don Alejo y la dueña del burdel "chocan sus vasos". Como recordará el lector de la novela, la Japonesita es el inesperado fruto de la noche de la apuesta, cuando la Japonesa Grande y la Manuela le ganan a don Alejandro Cruz.

\section{Sobre la anotación de la novela}

Mediante la anotación, se ha buscado transparentar las decisiones que subyacen al texto establecido para la presente edición, informando al lector sobre las diferencias de éste en relación con la edición princeps (Mortiz: 1966) y con la última que suponemos fue revisada por el propio Donoso (Alfaguara: 1995). De igual forma, advertimos acerca de las erratas identificadas en las dos ediciones que emplearon para el establecimiento del texto, así como de los momentos específicos en los que la novela ha sido 
fijada a partir de los materiales de archivo del escritor. Asimismo, ofrecemos información valiosa y sucinta sobre algunos elementos constitutivos del mundo narrado con la esperanza de que enriquezcan la experiencia de lectura de la novela, tales como datos sobre las ciudades específicas de la Región del Maule, los productos propios de la producción vitivinícola de la zona, el desarrollo ferroviario en Chile, la flora y la fauna, la cocina criolla y las canciones populares.

Con la finalidad de apoyar al lector en el proceso de intelección del texto, en lo que se refiere a las particularidades del español de Chile, incluimos un conjunto de notas en las que se definen voces y expresiones idiomáticas, en su mayoría tomadas del Diccionario de uso del español de Chile de la Academia Chilena de la Lengua, además del voseo verbal chileno, sus peculiaridades y denotaciones en la novela; la realización de estas últimas estuvo a cargo del Dr. Gonzalo Campos-Dintrans.

En el ámbito relacionado con la producción, edición, circulación y recepción del texto, se elaboraron notas que informan al lector de las alteraciones encontradas en otras ediciones que no corresponden a variantes de autor, sino a intromisiones editoriales. También, se incluyeron notas que, en momentos puntuales, hacen referencia a cómo ha sido leído un elemento específico por parte de la crítica, y otras que llaman la atención del lector sobre coincidencias con los dos textos de Donoso escritos en la década del sesenta, a saber, Este Domingo y El obsceno pájaro de la noche.

\section{El lugar del archivo en esta edición más allá de la fijación del texto}

Como he comentado anteriormente, recurrimos al archivo del escritor para respaldar decisiones de edición en momentos críticos, relacionadas con la fijación del texto. Por críticos entiendo aquellos en los que el cotejo sólo dejaba en evidencia diferencias sustanciales, pero no definía el camino a seguir para el añorado establecimiento textual más cercano a la última voluntad del escritor, meta de una edición crítica, independiente de sus particularidades y del proyecto editorial en el que se inserte. Así, ante la ausencia de un fichero informático o de otros tipos de paratextos que ayudaran eventualmente a rescatar los cambios hechos por José Donoso o por lo menos aprobados en una edición supervisada por él, los borradores fueron el bastión en el cual descansó la fijación textual.

La correspondencia del escritor, por su parte, constituyó una fuente invaluable en la reconstrucción de la historia del texto, de su producción y circulación, permitiendo disipar dudas, responder interrogantes y rellenar los vacíos que el cotejo y la revisión de la crítica textual $a b$ ovo iban, de manera irremediable, dejando en el camino. Entre las diversas preguntas que surgieron durante la identificación de las diferentes copias materiales de la novela y el consecutivo reconocimiento de las sucesivas ediciones y reediciones, resultó interesante que la novela hubiera tardado trece años en salir en 
Seix Barral (1979), considerando no sólo que para el propio Donoso ésta conformaba el "principal órgano de la literatura latinoamericana" (1998: 126), sino que, a partir de 1975, ya circulaban tres ediciones de la novela en España: Euros (1975), Bruguera (1977) y Sedmay (1977). Del mismo modo, determinar cuál fue el origen de la confusión de la crítica en torno a la fecha de la primera edición de la novela, citada en ocasiones con el año de 1965 y en otras con el de 1966. En ambos casos, el recurso que nos permitió hacer frente a estas interrogantes fue el intercambio epistolar con la Agencia Literaria Carmen Balcells, resguardada en el archivo de la Universidad de Princeton. Como discuto en la sección "Historia del texto", la censura bajo el régimen de Franco en España - cuando Donoso deseaba publicar la novela - en conjunto con los intereses comerciales de Seix Barral explican el porqué de la tardanza de la aparición de la novela en la editorial barcelonesa cuando ya no contaba con Carlos Barral (1979), a la vez que los requisitos impuestos por Sedmay en relación con el año (1965) que debía acompañar al copyright de los ejemplares impresos, tanto en Sedmay como en Bruguera.

\section{Palabras de cierre}

Para finalizar, una breve reflexión en torno al porqué de la selección de esta novela como el bastión para representar la producción de José Donoso, uno de los escritores que no podía faltar dentro de esa tradición literaria nacional que la Colección Biblioteca Chilena esboza desde el 2008. En la trayectoria de la obra y poética donosianas, El lugar sin límites constituye un hito. Para empezar, es su primera novela publicada fuera de Chile y en pleno Boom, así como la que divide, en términos de desarrollo, su producción, "earlier and later works", en palabras de Sharon Magnarelli (67). En segundo lugar, y desde el punto de vista del oficio de la escritura, este texto constituye el receso productivo y el laboratorio creativo que permitió a Donoso retomar, tiempo después, la escritura de su obra magna, El obsceno pájaro de la noche. Como pretendo demostrar en mi libro en curso, fosé Donoso escritor de $\mathrm{El}$ obsceno pájaro de la noche, es durante el proceso compositivo de El lugar sin límites cuando Donoso trabaja y logra ejecutar una serie de técnicas narrativas que, aplicadas posteriormente a El último Azcoitía-Obsceno pájaro de la noche, posibilitan la añorada culminación de la novela, convirtiendo a El lugar sin límites en una piedra angular dentro del desarrollo y construcción de esa poética y escritura de Donoso, que llega a un clímax en 1970 con la publicación de su novela más ambiciosa en términos de experimentación formal, El obsceno pájaro de la noche. En efecto, varias notas en la edición destacan elementos coincidentes en la tríada del sesenta (El lugar sin límites, Este domingo y El obsceno pájaro de la noche) que hacen eco de ese contacto/continuidad creativo; por ejemplo, el dolor de estómago que paraliza tanto al personaje Pancho Vega de El lugar sin límites como a Humberto 
Peñaloza en El obsceno pájaro de la noche (nota 84), o bien, los "alfeñiques" que Doña Blanca guarda en un tarro de té Mazawatte en El lugar sin límites y el abuelo/"La Muñeca" en Este Domingo (nota 197).

En tercer lugar, y ahora mirando el lugar que esta novela ocupa en la recepción de la obra donosiana, El lugar sin límites es, a todas luces, una de las que más ha acaparado la atención de la crítica. Como se puede constatar en la bibliografía elaborada en colaboración con Daniela Buksdorf (Donoso, 2020: 341-347), a más de cinco décadas del primer estudio centrado en la novela (1967), esta obra, de la mano de su protagonista - la Manuela - , sigue generando lecturas críticas en pleno siglo xxi. La Dra. Lorena Amaro, en "La historia no contada de Chile" (texto originalmente leído el día del lanzamiento para presentar la edición en Santiago), advierte cómo en los textos más actuales que contribuyen al discurso crítico de la novela se puede

observar la importancia de los contextos productivos de la escritura y la necesidad crítica de establecer lecturas estético-políticas en diálogo con los marcos históricos y sociales, al mismo tiempo que se revela, con el transcurso del tiempo, el avance de la escritura académica, que hasta cierto punto se distancia del ensayismo literario de las décadas anteriores.

Esperamos que este volumen publicado recientemente en Ediciones Universidad Alberto Hurtado llegue a la mayor cantidad de lectores posible y que ponga El lugar sin límites - una de las principales novelas de José Donoso - a disposición no sólo de sus fieles lectores, sino también de nuevas generaciones. Que el trabajo ecdótico realizado con la finalidad de rescatar este texto fundamental permita fomentar su (re)lectura, contribuyendo a mantener y renovar la discusión e interpretación de una pieza maestra tanto de la literatura chilena como del acervo latinoamericano.

\section{Bibliografía}

AMARo, Lorena

"La historia no contada de Chile", en Santiago (8 de enero de 2020). Consultado en: http:// revistasantiago.cl/literatura/la-historia-no-contada-de-chile/ [07/10/2019].

Delgado, Josefina

"La mirada sin cuerpo", en Cuadernos Hispanoamericanos, número 634 (abril de 2003), 21-29.

Donoso, José

El lugar sin límites. México: Joaquín Mortiz, 1966.

El lugar sin límites. Barcelona: Bruguera, 1977.

El lugar sin límites. Barcelona: Seix Barral, 1979. 
El lugar sin límites. Santiago: Alfaguara, 1995.

Historia personal del "boom”. Madrid: Alfaguara, 1998.

El lugar sin límites. Edición crítica de María Laura Bocaz Leiva. Santiago: Ediciones Universidad Alberto Hurtado, 2020 (Colección Biblioteca Chilena).

Notebook 32. Fosé Donoso Papers. Department of Rare Books and Special Collections, University of Iowa.

"Second Draft". Tss. Fosé Donoso Papers. Department of Rare Books and Special Collections, University of Iowa.

MAGNARELLI, Sharon

Understanding fosé Donoso. South Carolina: University of South Carolina Press, 1992.

"El lugar sin límites. Límites, centros y discurso". Traducción de Ángela San Martín Vásquez, en José Donoso. El lugar sin límites. Edición crítica de María Laura Bocaz Leiva. Santiago: Ediciones Universidad Alberto Hurtado, 2020 (Colección Biblioteca Chilena).

MejÍA, Eduardo

"Volar alto", en La Gaceta del Fondo de Cultura Económica, número 413 (2005), 4-6.

Moreno Turner, Fernando

"La inversión como norma. A propósito de El lugar sin límites", en Cuadernos Hispanoamericanos, número 295 (1975), 19-42.

Ostrov, Andrea

"Espacio y sexualidad en El lugar sin límites de José Donoso", en Revista Iberoamericana, volumen 65, número 187 (1999), 341-348.

Patemain, Alejandro

"El infierno de la ambigüedad", en Temas. Revista de Cultura, volumen 12 (mayo-junio de 1967), 44-48.

Rodríguez Monegal, Emir

"El mundo de José Donoso", en Mundo Nuevo, número 12 (1967), 77-85.

SARDUY, Severo

"Escritura/travestismo", en Mundo Nuevo, número 20 (febrero de 1968), 72-74.

Sifuentes-Jáuregui, Ben

"Gender without Limits: Transvestism and Subjectivity in El lugar sin limites", en Daniel Balderston and Donna J. Guy (editors). Sex and Sexuality in Latin America. New York: New York University Press, 1997, 44-61.

140 (an)ecdótica * vol. IV, núm. 2, julio-diciembre 2020 